\title{
Recognition of a Bacterial Isolate from "Rhizosphere" of a Tea Plant from a Tea Garden of Barak Valley, Assam (India).
}

\author{
${ }^{1 *}$ Mrinal Kanti Bhattacharjee, P.B.Mazumder ${ }^{2}$ and ${ }^{3}$ G.D.Sharma. \\ ${ }^{1 *}, 2$ Department of Biotechnology, Assam University, Silchar, Assam. \\ ${ }^{3}$ Bilaspur University.
}

\begin{abstract}
A bacterium was detached from the "rhizosphere" part of tea plant from a tea garden of Barak Valley, Assam (India). The bacterial isolate was separated on an " $N$-free medium" and it was identified on the basis of its "external attributes". The "Gram staining" and "molecular features" were also conceded in. Further the molecular work through "16SrDNA scrutiny" of the isolate was decided out and on the foundation of that; "species level" identification of that bacterium was made. The "evolutionary tree" was constructed by taking the concerned bacterial isolate that was marked as "Azo-4"with taking ten other close strains that were available in the "NCBI" GenBank nucleotide database.
\end{abstract}

Key words: external attributes, detached, 16SrDNA scrutiny, species level.

\section{Introduction:}

The "nitrogen" present in the atmosphere in massive amount as a gas and it gets reduced to some reducible forms of organic compounds through "biological reactions" with the help of microbes of prokaryotic nature [6]. As free living bacteria and cyanobacteria desire to live in a broad range of habitats with a varying degree of nutrients, oxygen, $\mathrm{pH}$, etc [6]. Possessing of highest respiratory rates the "Azotobacters" are "aerobic bacteria" [6], as the members from this group happens to be "mesophilic bacteria" that have a preference to grow at temperature of around $30^{\circ} \mathrm{C}$ [6]. It is been quit known that "microbes" hardly does any harm to the "environment", hence for better and safer agricultural outputs, microbes are thought to be a secured and a safer path.

This paper encompasses with a free living nitrogen fixing bacteria marked as "AZO-4" isolated from tea "rhizosphere" of a tea plant from a tea garden of Barak Valley, Assam (India).Some of the characteristics features of the isolate have been presented in the current paper. The scrutiny by "16SrDNA" for the bacterial isolate was also been made and then results were represented nicely.

\section{Material and Methods:}

Soil from the "rhizosphere" zone of tea plant was used for the separation of the bacterial isolate; the media chosen was "Ashby sucrose" medium [9] for isolating the bacterial isolate. The "Petri Plate" was brewed for almost a week at room temperature. Afterwards on recurring streaking on the identical medium "pure colony" was attained.

\section{Morphology for the bacterial isolate:}

The pure isolate marked as "AZO-4" was examined for colony form and shape on the same medium as per the procedures described by [2] also the further again morphology is ascertained by procedures described, as per [4]."Gram staining" was performed by following the method described in the "Practical laboratory Manual of Microbiology" by Dubey et al. (2002) [5].

\section{"16SrDNA" Assessment:}

By a standard scheme the genomic DNA was extracted from the isolate (AZO-4).After that from the isolated DNA, by using PCR a fragment of "16S rDNA" was allowed for amplification. After removing all the contaminants the PCR amplicon was thus purified. With the help of "8F" and "1492R" "primers" [7]; [3] the forward and the reverse DNA sequencing reaction of that purified PCR amplicon was made nicely. After that from the forward and reverse sequence data a "consensus sequence" of 1248bp of "16S rDNA" gene was created with the aid of "aligner software". In the next step, with that 16SrDNA gene sequence the BLAST [1] was carried out by making use of nrdatabase of NCBI (www.ncbi.nlm.nih.gov) GenBank database. Then, on the basis of the maximum identity score, first ten sequences were made to get selected and thereafter the sequences were made to get aligned with the help of the "multiple alignments" software program Clustal W [14].Further on generating distance matrix and then by using the MEGA4 [13] was utilized for generating the phylogenetic relationship, by following the Neighbor-joining method as being described by [12] and the Kimura's twoparameter nucleotide distances [11], as from 500 replicates the "bootstrap consensus" tree was concluded [8]. 
The "16SrDNA" sequence that has been obtained for the bacterial isolate (Azo-4) along with close ten strains that are available in the NCBI GenBank nucleotide database using those, the "phylogenetic tree" was build. The whole process was carried at Xcelris Labs.Ltd (Ahmedabad).

The sequencing Primers used were:

8F (AGAGTTTGATCCTGGCTCAG) - forward primer.

1492R (GGTTACCTTGTTACGACTT) - reverse primer

Morphology and Gram Staining result for the Isolate:

\section{Results:}

The morphology reveals that the isolate marked as "AZO-4" is round, circular in shape seen in "Petri plate". From the "Gram negative", rod shaped bacteria when seen under microscope.

"16SrDNA" Assessment Result:

The result of"16SrDNA" analysis, from "nucleotide" homology" and "phylogenetic" investigation of that strain, it has been come to know that the bacterial isolate marked as "Azo-4" is being identified as Azotobacter beijerinckii strain ICMP8673. The sequence of that above mentioned bacterial strain (Azo-4) on doing BLAST showed homology with current bacterial isolate "Azotobacter beijerinckii strain ICMP 8673" which had an accession number EF100152.1.On submitting the current strain of the bacterial strain "Azo-4"to NCBI, an accession number KC 172855 was assigned. A "Phylogenetic tree" was built eventually based on comparing the 16SrDNA sequence of that bacterial strain (Azo-4) along with the other close ten strains that were available in the GenBank nucleotide database of NCBI.

Sequence producing significant Alignment (given below in the table) (carried out at Xcelris labs)

\begin{tabular}{|l|l|l|l|l|l|l|}
\hline Accession & Description & $\begin{array}{l}\text { Max } \\
\text { Score }\end{array}$ & $\begin{array}{l}\text { Total } \\
\text { Score }\end{array}$ & $\begin{array}{l}\text { Query } \\
\text { coverage }\end{array}$ & $\underline{\text { Evalue }}$ & $\underline{\text { Max ident }}$ \\
\hline GU372346.1 & Beijerinckia indica strain SDSA-I30/2 & $\mathbf{2 3 0 5}$ & $\mathbf{2 3 0 5}$ & $\mathbf{1 0 0 \%}$ & $\mathbf{0 . 0}$ & $\mathbf{1 0 0 \%}$ \\
\hline EF100152.1 & Azotobacter beijerinckii strain ICMP 8673 & $\mathbf{2 3 0 5}$ & $\mathbf{2 3 0 5}$ & $\mathbf{1 0 0 \%}$ & $\mathbf{0 . 0}$ & $\mathbf{1 0 0 \%}$ \\
\hline EF100151.1 & Azotobacter beijerinckii strain ICMP 4032 & $\mathbf{2 3 0 5}$ & $\mathbf{2 3 0 5}$ & $\mathbf{1 0 0 \%}$ & $\mathbf{0 . 0}$ & $\mathbf{1 0 0 \%}$ \\
\hline NR_042071.1 & Azotobacter beijerinckii & $\mathbf{2 3 0 5}$ & $\mathbf{2 3 0 5}$ & $\mathbf{1 0 0 \%}$ & $\mathbf{0 . 0}$ & $\mathbf{1 0 0 \%}$ \\
\hline AB429526.1 & Azotobacter beijerinckii, strain: C4 & $\mathbf{2 2 7 6}$ & $\mathbf{2 2 7 6}$ & $\mathbf{1 0 0 \%}$ & $\mathbf{0 . 0}$ & $\mathbf{9 9 \%}$ \\
\hline GQ397081.1 & Uncultured bacterium clone AK4DE2_06E & $\mathbf{2 2 7 2}$ & $\mathbf{2 2 7 2}$ & $\mathbf{1 0 0 \%}$ & $\mathbf{0 . 0}$ & $\mathbf{9 9 \%}$ \\
\hline JN641801.1 & Azotobacter chroococcum strain A5 & $\mathbf{2 1 9 8}$ & $\mathbf{2 1 9 8}$ & $\mathbf{1 0 0 \%}$ & $\mathbf{0 . 0}$ & $\mathbf{9 8 \%}$ \\
\hline EU930421.1 & Azotobacter chroococcum strain 10006 & $\mathbf{2 1 9 8}$ & $\mathbf{2 1 9 8}$ & $\mathbf{1 0 0 \%}$ & $\mathbf{0 . 0}$ & $\mathbf{9 8 \%}$ \\
\hline EF620440.1 & Azotobacter chroococcum strain ISSDS-397 & $\mathbf{2 1 9 8}$ & $\mathbf{2 1 9 8}$ & $\mathbf{1 0 0 \%}$ & $\mathbf{0 . 0}$ & $\mathbf{9 8 \%}$ \\
\hline EF100153.1 & Azotobacter chroococcum strain ICMP 4031 & $\mathbf{2 1 9 8}$ & $\mathbf{2 1 9 8}$ & $\mathbf{1 0 0 \%}$ & $\mathbf{0 . 0}$ & $\mathbf{9 8 \%}$ \\
\hline
\end{tabular}

Distance Matrix (is given below) (carried out at Xcelris labs):

\begin{tabular}{|c|c|c|c|c|c|c|c|c|c|c|c|c|}
\hline AZO-4 & 1 & & 0.000 & 0.000 & 0.000 & 0.000 & 0.001 & 0.002 & 0.003 & 0.003 & 0.003 & 0.003 \\
\hline GU372346.1 & 2 & 0.000 & & 0.000 & 0.000 & 0.000 & 0.001 & 0.002 & 0.003 & 0.003 & 0.003 & 0.003 \\
\hline EF100152.1 & 3 & 0.000 & 0.000 & & 0.000 & 0.000 & 0.001 & 0.002 & 0.003 & 0.003 & 0.003 & 0.003 \\
\hline EF100151.1 & 4 & 0.000 & 0.000 & 0.000 & & 0.000 & 0.001 & 0.002 & 0.003 & 0.003 & 0.003 & 0.003 \\
\hline NR_042071.1 & 5 & 0.000 & 0.000 & 0.000 & 0.000 & & 0.001 & 0.002 & 0.003 & 0.003 & 0.003 & 0.003 \\
\hline AB429526.1 & 6 & 0.002 & 0.002 & 0.002 & 0.002 & 0.002 & & 0.002 & 0.003 & 0.003 & 0.003 & 0.003 \\
\hline GQ397081.1 & 7 & 0.005 & 0.005 & 0.005 & 0.005 & 0.005 & 0.006 & & 0.003 & 0.003 & 0.003 & 0.003 \\
\hline JN641801.1 & 8 & 0.010 & 0.010 & 0.010 & 0.010 & 0.010 & 0.008 & 0.015 & & 0.000 & 0.000 & 0.000 \\
\hline EU930421.1 & 9 & 0.010 & 0.010 & 0.010 & 0.010 & 0.010 & 0.008 & 0.015 & 0.000 & & 0.000 & 0.000 \\
\hline EF620440.1 & 10 & 0.010 & 0.010 & 0.010 & 0.010 & 0.010 & 0.008 & 0.015 & 0.000 & 0.000 & & 0.000 \\
\hline EF100153.1 & 11 & 0.010 & 0.010 & 0.010 & 0.010 & 0.010 & 0.008 & 0.015 & 0.000 & 0.000 & 0.000 & \\
\hline
\end{tabular}

The Matrix is given in the above table the ten isolates with accession numbers are given along with the Azo-4 isolate.

Sequence of Azo-4 got by forward primer (8F) (836 bp):

TACGTCCTACGGGAGAAAGTGGGGGCTCTTCGGACCTCACGCTATCGGATGAGCCTAGGTCGGATT AGCTAGTTGGTGGGGTAAAGGCCCACCAAGGCGACGATCCGTAACTGGTCTGAGAGGATGATCAG TCACACTGGAACTGAGACACGGTCCAGACTCCTACGGGAGGCAGCAGTGGGGAATATTGGACAAT GGGCGAAAGCCTGATCCAGCCATGCCGCGTGTGTGAAGAAGGTCTTCGGATTGTAAAGCACTTTA AGTTGGGAGGAAGGGCTGTAAGTTAATACCTTGCAGTTTTGACGTTACCGACAGAATAAGCACCG 
Recognition of a Bacterial Isolate from "Rhizosphere" of a Tea Plant from a Tea Garden of Barak

GCTAACTTCGTGCCAGCAGCCGCGGTAATACGAAGGGTGCAAGCGTTAATCGGAATTACTGGGCG TAAAGCGCGCGTAGGTGGTTTGGTAAGTTGGATGTGAAAGCCCCGGGCTCAACCTGGGAACTGCA TCCAAAACTGCCTGACTAGAGTACGGTAGAGGGTGGTGGAATTTCCTGTGTAGCGGTGAAATGCG TAGATATAGGAAGGAACACCAGTGGCGAAGGCGACCACCTGGACTGATACTGACACTGAGGTGCG AAAGCGTGGGGAGCAAACAGGATTAGATACCCTGGTAGTCCACGCCGTAAACGATGTCGACTAGC CGTTGGGCTCCTTGAGAGCTTAGTGGCGCAGCTAACGCATTAAGTCGACCGCCTGGGGAGTACGG CCGCAAGGTTAAAACTCAAATGAATTGACGGGGGCCCGCACAAGCGGTGGAGCATGTGGTTTAAT TCGAAGCAACGCGAAGAACCTTACCTGGCCTTGACATCCTGCGAACTGGGTAGAG

Sequence of Azo-4 got by reverse primer (1492R) (881 bp):

CAACCCACTCCCATGGTGTGACGGGCGGTGTGTACAAGGCCCGGGAACGTATTCACCGCGACATT CTGATTCGCGATTACTAGCGATTCCGACTTCACGCAGTCGAGTTGCAGACTGCGATCCGGACTACG ATCGGTTTTCTGGGATTAGCTCCGCCTCGCGACTTGGCAACCCTCTGTACCGACCATTGTAGCACGT GTGTAGCCCTGGCCGTAAGGGCCATGATGACTTGACGTCATCCCCACCTTCCTCCGGTTTGTCACC GGCAGTCTCCTTAGAGTGCCCACCATGACGTGCTGGTAACTAAGGACAAGGGTTGCGCTCGTTACG GGACTTAACCCAACATCTCACGACACGAGCTGACGACAGCCATGCAGCACCTGTCTCTGCGCTCCC GAAGGCACCCAGGTATCTCTACCCAGTTCGCAGGATGTCAAGGCCAGGTAAGGTTCTTC

GCGTTGCTTCGAATTAAACCACATGCTCCACCGCTTGTGCGGGCCCCCGTCAATTCATTTGAGTTTT AACCTTGCGGCCGTACTCCCCAGGCGGTCGACTTAATGCGTTAGCTGCGCCACTAAGCTCTCAAGG AGCCCAACGGCTAGTCGACATCGTTTACGGCGTGGACTACCAGGGTATCTAATCCTGTTTGCTCCC CACGCTTTCGCACCTCAGTGTCAGTATCAGTCCAGGTGGTCGCCTTCGCCACTGGTGTTCCTTCCTA TATCTACGCATTTCACCGCTACACAGGAAATTCCACCACCCTCTACCGTACTCTAGTCAGGCAGTTT TGGATGCAGTTCCCAGGTTGAGCCCGGGGCTTTCACATCCAACTTACCAAACCACCTACGCGCGCT TTACGCCCAGTAATTCCGATTAACGCT

Consensus Sequence of Azo-4 (1248 bp):

TACGTCCTACGGGAGAAAGTGGGGGCTCTTCGGACCTCACGCTATCGGATGAGCCTAGGTCGGATT AGCTAGTTGGTGGGGTAAAGGCCCACCAAGGCGACGATCCGTAACTGGTCTGAGAGGATGATCAG TCACACTGGAACTGAGACACGGTCCAGACTCCTACGGGAGGCAGCAGTGGGGAATATTGGACAAT GGGCGAAAGCCTGATCCAGCCATGCCGCGTGTGTGAAGAAGGTCTTCGGATTGTAAAGCACTTTA AGTTGGGAGGAAGGGCTGTAAGTTAATACCTTGCAGTTTTGACGTTACCGACAGAATAAGCACCG GCTAACTTCGTGCCAGCAGCCGCGGTAATACGAAGGGTGCAAGCGTTAATCGGAATTACTGGGCG TAAAGCGCGCGTAGGTGGTTTGGTAAGTTGGATGTGAAAGCCCCGGGCTCAACCTGGGAACTGCA TCCAAAACTGCCTGACTAGAGTACGGTAGAGGGTGGTGGAATTTCCTGTGTAGCGGTGAAATGCG TAGATATAGGAAGGAACACCAGTGGCGAAGGCGACCACCTGGACTGATACTGACACTGAGGTGCG AAAGCGTGGGGAGCAAACAGGATTAGATACCCTGGTAGTCCACGCCGTAAACGATGTCGACTAGC CGTTGGGCTCCTTGAGAGCTTAGTGGCGCAGCTAACGCATTAAGTCGACCGCCTGGGGAGTACGG CCGCAAGGTTAAAACTCAAATGAATTGACGGGGGCCCGCACAAGCGGTGGAGCATGTGGTTTAAT TCGAAGCAACGCGAAGAACCTTACCTGGCCTTGACATCCTGCGAACTGGGTAGAGATACCTGGGT GCCTTCGGGAGCGCAGAGACAGGTGCTGCATGGCTGTCGTCAGCTCGTGTCGTGAGATGTTGGGTT AAGTCCCGTAACGAGCGCAACCCTTGTCCTTAGTTACCAGCACGTCATGGTGGGCACTCTAAGGAG ACTGCCGGTGACAAACCGGAGGAAGGTGGGGATGACGTCAAGTCATCATGGCCCTTACGGCCAGG GCTACACACGTGCTACAATGGTCGGTACAGAGGGTTGCCAAGTCGCGAGGCGGAGCTAATCCCAG AAAACCGATCGTAGTCCGGATCGCAGTCTGCAACTCGACTGCGTGAAGTCGGAATCGCTAGTAAT CGCGAATCAGAATGTCGCGGTGAATACGTTCCCGGGCCTTGTACACACCGCCCGTCACACCATGGG AGTGGGTTG

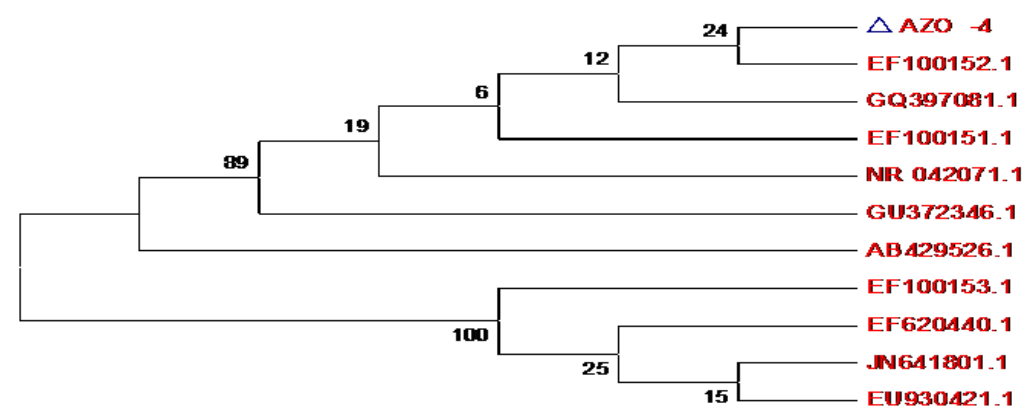

Figure: 1.Azo-4 evolutionary relationship with 10 other bacterial isolates, $\mathbf{1 0}$ isolate sequences are taken from NCBI, GenBank database. (The whole method done at Xcelris labs) 


\section{Discussion:}

It has been come under consideration, the isolate was seen as rod shape under microscope .Accordingly from the results of "Gram staining", it has been seen that the isolate is of "Gram" negative type.

The above check of the isolate Azo-4 was compared with the results of Joseph et al. (2007) [10] for their similar isolates of Azotobacter and it was found that, the isolates showed similar result as the similar isolates of Azotobacter isolated by Joseph et al. (2007) were found to be seen as Gram negative by nature and rod shape in appearance when seen under microscope as of [10].Finally the isolate (Azo-4) was made to get identified and a GenBank Accession Number KC172855 has been provided by GenBank of NCBI for that isolate. Lastly, "phylogenetic tree" was set up.

\section{Conclusion:}

Clearly the study has brought a fascinating move in this part of Barak Valley, Assam (India).As on this trial an isolate was isolated and identified on basis of 'external attribute' and 'molecular' implementation by "16SrDNA".

\section{Acknowledgements:}

We are grateful to NCBI (www.ncbi.nlm.nih.gov) for providing us with an accession number for that bacterial isolate. We also show deep thanks to different (references) sources from where 'knowledge' about the topic was obtained. Also we express thanks to those persons who were involved for helping us directly or indirectly during the entire study, and more importantly to Xcelris labs. Ltd, Ahmedabad for their utmost cooperation in "sequencing" and "phylogenetic" work. Also, show thanks towards the parent institution Assam University for giving the support in all sorts. Finally, I thank everyone right from the beginning towards the end who helped us in carrying out the work.

\section{References:}

[1]. Altschul, S.F., Gish, W., Miller, W., Myers, E.W.and Lipman, D.J. (1990).Basic Local Alignment Search Tool. Journal of Molecular Biology.215:403-410.

[2]. Anonymous. (1957).Manual of Microbiological Methods. McGraw Hill Book Company Inc. New York. p.127.

[3]. Anonymous. (2010). "Universal bacterial Identification by PCR and DNA sequencing of 16SrRNA gene".PCR for Clinical Microbiology.Part3:209-214.

[4]. Cappuccino, J.G.and Sherman, N. (2005).Microbiology: A Laboratory Manual.7 $7^{\text {th }}$ edition. The Pearson Education. Darling Kindersley (Ind) Pvt.

[5]. Dubey, R.C. and. Maheshwari, D.K. (2002).A Textbook of Practical Microbiology.S.Chand Co.Ltd, Ram Nagar.Newdelhi.p.352.

[6]. Dubey, R.C. (2004). "Biological nitrogen fixation". A Textbook of Biotechnology.S.Chand and Company Ltd. New Delhi. p 202216.

[7]. Eden, P.E., Schmidt, T.M., Blakemore, R.P.and Pace, N.R. (1991). "Phylogenetic analysis of Aquaspirillium magnetotacticum using PCR-amplified 16SrRNA-Specific DNA". International Journal of Systematic Bacteriology.41 (2):324-325.

[8]. Felsenstein, J. (1985).Confidence limits on phylogenies: An approach using the bootstrap. Evolution 39:783-791.

[9]. Jimenez, D.J., Montana, J.S. and Martinez, M.M. (2011).Characterization of Free Nitrogen Fixing Bacteria of genus Azotobacter in Organic Vegetable- Grown Colombian Soils. Brazilian Journal of Microbiology.42:846-858.

[10] Joseph, B., Ranjan Patra, R. and Lawrence, R. (2007). Characterization of "plant growth promoting rhizobacteria" associated with chickpea (Cicer arietinum L.).International Journal of plant production.1 (2):141-152.

[11]. Kimura, M. (1980). A simple method for estimating evolutionary rates of base substitutions through comparative studies of nucleotide sequences. Journal of Molecular Evolution.16:111-120.

[12]. Saitou, N. and Nei, M. (1987). The neighbor-joining method: A new method for reconstructing phylogenetic trees. Molecular Biology and Evolution.4:406-425.

[13]. Tamura, K., Dudley, J., Nei, M. and Kumar, S. (2007). MEGA4: Molecular Evolutionary Genetics Analysis (MEGA) software version 4.0. Molecular Biology and Evolution.24:1596-1599.

[14]. Thompson, J.D., Higgins, D.G. and Gibson, T.J. (1994) .CLUSTAL W: improving the sensitivity of progressive multiple sequence alignment through sequence weighting, position-specific gap penalties and weight matrix choice. Nucleic Acids research.22 (22):4673-4680. 